RELATO DE CASO

\section{COMPLEXO OEIS - RARA ASSOCIAÇÃO DE ANOMALIAS CONGÊNITAS, EM PALMAS - TOCANTINS}

OEIS COMPLEX - RARE ASSOCIATION OF CONGENITAL ANOMALIES, IN PALMAS - TOCANTINS

Edilberto Vasconcelos Pereira Júnior ${ }^{1}$, lago Assunção Pereira², Igor Assunção Pereira ${ }^{2}$, Milena Borges de Castro ${ }^{1}$, Vitoria Aguiar de Faria ${ }^{1}$, Elizete Ferreira Leite ${ }^{3}$.

\section{RESUMO}

Introdução: O complexo OEIS é uma combinação de malformações congênitas graves incluindo onfalocele, extrofia de cloaca, ânus imperfurado e defeitos espinhais. É extremamente raro, com ocorrência estimada em 1: 200.000 a 1: 400.000 gestações. A maioria dos casos são esporádicos e com etiologia incerta e possivelmente heterogênea. Caso clínico: Descrito o caso de um bebê nascido de parto cesáreo com 37 semanas de gestação, que apresentou malformação abdominal em ultrassonografia obstétrica. Ao nascimento, apresentava múltiplas malformações: onfalocele, genitália ambígua, extrofia de bexiga e cloaca, ânus imperfurado e angulação espinhal. Apresenta cariótipo 46, XY. Sendo submetido a intervenção cirúrgica subsequente. Discussão e considerações finais: o caso descrito atende aos critérios clínicos do complexo OEIS, somando-se ainda anormalidade genital. Apresenta na história pré-natal, mãe com Diabetes Mellitus Gestacional, possível fator de risco para extrofia de cloaca. O prognóstico do paciente portador do Complexo de OEIS depende da severidade das anormalidades apresentadas e da realização de um pré-natal adequado. 0 seguimento é complicado e requer abordagem multidisciplinar. Dentre as complicações incluem-se principalmente infecções de repetição, além de possíveis problemas de cunho psicossociais.

Palavras-chave: Onfalocele. Extrofia de cloaca. Complexo OEIS. Anormalidades. Pré-natal.

\begin{abstract}
Introduction: Introduction: The OEIS complex is a combination of severe congenital malformations including omphalocele, cloacal exstrophy, imperforate anus, and spinal defects. It is extremely rare, with an estimated occurrence of 1 : 200,000 to 1: 400,000 pregnancies. Most cases are sporadic and with uncertain and possibly heterogeneous etiology. Case report: Described the case of a baby born with cesarean delivery at 37 weeks of gestation, who presented abdominal malformation on obstetric ultrasonography. At birth, he had multiple malformations: omphalocele, ambiguous genitalia, exstrophy of the bladder and cloaca, imperforate anus and spinal angulation. It presents karyotype $46, \mathrm{XY}$. Being submitted to subsequent corrective surgeries. Discussion and conclusion: the case described meets the clinical criteria of the OIES complex, adding to the genital abnormality. Presents in the prenatal history, mother with Gestational Diabetes, possible risk factor for cloacal exstrophy. The prognosis of the patient with the OEIS complex depends on the severity of the abnormalities presented and the performance of an adequate prenatal care. Follow-up is complicated and requires a multidisciplinary approach. Complications include mainly recurrent infections, as well as possible psychosocial problems.worldwide.
\end{abstract}

Keywords: Omphalocele. Cloacal exstrophy. OEIS complex. Abnormalities. Prenatal.
Direitos Autorais: C 2018 Pereira Júnior et al. Este é um artigo de acesso aberto que permite o uso, a distribuição e a reprodução sem restrições em qualquer meio, desde que o autor original e a fonte sejam creditados.

Conflito de interesses: os autores declararam que não existem conflitos de interesses. 


\section{INTRODUÇÃO}

O complexo OEIS é uma combinação de malformações congênitas graves incluindo onfalocele, extrofia de cloaca, ânus imperfurado e defeitos espinhais, primeiramente descrito com essa terminologia em 1978, por Carey et $\mathrm{al}^{1}$. É extremamente raro, com ocorrência estimada em 1: 200.000 a 1: 400.000 gestações $^{2}$, podendo a sua ocorrência ser subestimada, por não ser muitas vezes identificada em caso de abortos ou natimortos ${ }^{3}$.

A maioria dos casos são esporádicos, com etiologia incerta mas possivelmente heterogênea. Propõe-se que a patogênese decorra de uma falha de migração do mesênquima caudal resultando na persistência da cloaca e anus imperfurado ${ }^{4}$. Há relatos de sua recorrência em familiares e em gêmeos monozigóticos ${ }^{5}$, sugerindo um componente genético na patogênese da síndrome ${ }^{6}$. Contudo, também há relatos de gêmeos monozigóticos discordantes para a síndrome, sugerindo um componente etiológico não genético, dentre eles temos: patogenia vascular uterina, insuficiência vascular uteroplacentária, fertilização in vitro, traumas, uso de substâncias ${ }^{5}$.

Muitos casos não são detectados no pré-natal e na maioria das vezes há perda fetal. O diagnóstico pré-natal é de difícil realização e pode ser sugerido por meio de uma ultrassonografia pré-natal com presença de defeitos espinhais e defeitos na parede abdominal do feto. A síndrome pode ser associada a outros defeitos ocasionais como anormalidades renais, defeitos na formação de extremidade e anormalidades genitais $^{6,7,8}$.

O seguimento pós-natal é exigente, necessitando de abordagem cirúrgica nas primeiras horas de vida com equipe multidisciplinar, múltiplas abordagens cirúrgicas e seguimento multiprofissional ao longo da vida.

Nos últimos 20 anos a taxa de sobrevivência entre os pacientes que apresentam extrofia de cloaca tem passado de $90 \%{ }^{9}$, o seguimento do paciente é multidisciplinar na tentativa de minimizar o impacto severo na qualidade de vida.

\section{DESCRIÇÃO DO CASO}

M.T.C. nascido de parto cesáreo em novembro de 2016 no Hospital e Maternidade Dona Regina em Palmas - TO. Mãe com ultrassonografia (USG) obstétrica prévia com idade gestacional (IG) de 30 semanas apresentando defeito de fechamento de parede abdominal anterior, sugerindo gastrosquise. Pais não consanguíneos. Mãe na 5 a gestação, com 2 partos normais, 1 parto cesáreo e 1 aborto prévio, realizou 4 consultas de pré-natal com sorologias de 20 trimestre negativas, tipo sanguíneo B positivo, apresentou Diabetes Mellitus Gestacional, abordada com insulinoterapia. Negou etilismo, tabagismo, uso de teratógenos ou outros casos de malformação fetal na família. M.T.C nasceu à termo (IG de 37 semanas), pesando $3.480 \mathrm{~g}$, medindo $46 \mathrm{~cm}$, perímetro cefálico de $34 \mathrm{~cm}$ e apresentou Índice de Apgar no primeiro minuto de 5 e no quinto minuto de 9 . Ao nascimento, apresentava múltiplas malformações: onfalocele, genitália ambígua, extrofia de bexiga e cloaca, ânus imperfurado e angulação espinhal (Figura 1). Submetido à redução e contenção da onfalocele com placa de hidrocoloide ainda na sala de parto e mantido suporte clínico.

Ultrassonografia transfontanelar apresentou hipoplasia do esplênio do corpo caloso. Ecocardiograma evidenciou forame oval patente de $1,7 \mathrm{~mm}$.

Ultrassonografia abdominal demonstrou fígado, vias biliares, pâncreas, baço sem anormalidade, rim esquerdo e direito em topografia habitual e forma, contorno, mobilidade, tamanho, ecogeneidade córtico-medular normais, com boa dissociação parênquima-sinusal; revelou ainda vesícula biliar com conteúdo hipoecóico; aspecto de baço ectópico situado em flanco esquerdo inferior ao rim ipsilateral, gases em estômago e alças instestinais, vasos cava inferior e aorta abdominal com trajeto, calibre e pulsatilidade normais e bexiga não visualizada.

Cariótipo mostrou ser 46, XY (sexo masculino); sorologias STORCH (Sífilis, Toxoplasmose, Rubéola, Citomegalovírus e Herpes) negativas e tipo sanguíneo $O$ positivo.

Realizado até os dois meses de idade ampicilina e gentamicina por 10 dias, cefepime por 14 dias, amicacina por 14 dias e micafungina por 14 dias.

Aos 2 meses de idade foi encaminhado ao Hospital da Criança no Rio de Janeiro-RJ, e submetido à segunda intervenção cirúrgica com fechamento da extrofia de bexiga, ressecção da atresia ileal, anastomose ileocecal e confecção de colostomia dupla-boca, sendo observados presença de cólon rudimentar e duplicado, além de falo intravesical. Após maturação cirúrgica, foi iniciado dieta enteral, até então em dieta parenteral devido às más-formações.

Permaneceu internado no Rio de Janeiro por cerca de 15 dias, ao exame de rastreio infeccioso se mostrou colonizado com Staphylococcus aureus resistente a oxacilina (MRSA), Acinetobacter baumannii multirresistente, Serratia produtora de beta-lactamase de espectro estendido (ESBL) e enterobactéria resistente aos carbapenêmicos (ERC). Realizado durante a internação piperacilina e tazobactan por 8 dias, oxacilina por 7 dias, amicacina por 7 dias, vancomicina por 17 dias e meropenen por 10 dias.

Evoluiu com bom estado clínico e boa aceitação da dieta enteral, com necessidade de acompanhamento fonoaudiológico para estímulo da sucção e antibioticoprofilaxia para infecção urinária e retorno programado em 12 meses para abordagem de genitália.

Um termo de consentimento livre e esclarecido foi assinado pela responsável do paciente, autorizando publicação do caso, assim como utilização da foto.

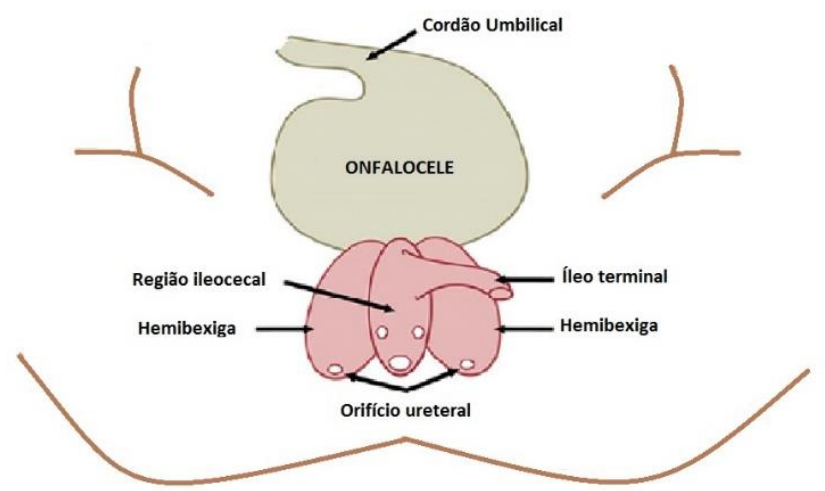

Figura 1. Representação de onfalocele e extrofia de cloaca. 


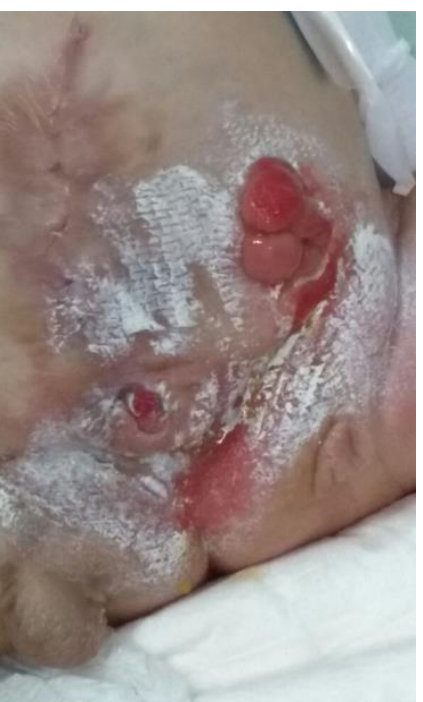

Figura 2. Foto aos 4 meses de idade, após correção cirúrgica. Apresentando áreas de dermatite tópica devido excreção urinária. Regiões brancas devido pomada protetora.

\section{DISCUSSÃO}

O paciente descrito neste relato de caso apresenta a clássica malformação do complexo OEIS, somando-se ainda a anormalidade de genitália. Como já citado, o complexo OEIS exibe etiologia desconhecida e possivelmente heterogênea, a extrofia de cloaca é relacionada com mutações em genes homeobox e receptores de ácido retinóico ${ }^{4}$, associada com obesidade materna ${ }^{4}$, exposição ao ácido valpróico ${ }^{10}$, uso de metanfetamina, tabagismo e Diabetes Mellitus ${ }^{4}$, este último apresentado pela progenitora do paciente. Contudo, essas associações estão limitadas a poucos casos e não há dados suficientes para vincular o complexo OEIS com quaisquer teratógenos específicos ${ }^{12}$.

O prognóstico do paciente portador do complexo de OEIS depende da severidade das anormalidades apresentadas e da realização de um pré-natal adequado. A identificação prénatal permite o nascimento em ambiente hospitalar de alta complexidade, necessário para o acolhimento adequado peri e pós-natal. Com a intervenção cirúrgica para correções, mesmo que parciais, das anormalidades e defeitos, a sobrevida desses pacientes tem melhorado de forma substancial e se somando a isso há meios de prevenção de complicações com medidas de suporte, como a antibioticoprofilaxia e a abordagem multidisciplinar.

O seguimento é complicado e requer, como já dito, abordagem multidisciplinar e multiprofissional. Dentre as complicações incluem-se principalmente infecções de repetição, além de possíveis problemas de cunho psicossociais ${ }^{3,10}$. O estresse psicológico e social a qual tais pacientes são submetidos são imensuráveis ${ }^{3}$. Um dos desafios é a própria construção de identidade gênero-corporal, especialmente para pacientes com cariótipo $46 \mathrm{XY}$, que não possuem tecido para uma reconstrução adequada ${ }^{10}$. Tais questões podem levar a dificuldades de desenvolvimento psicossexuais mais tarde na vida, ficando evidente a necessidade de um acompanhamento multiprofissional para lidar com todos os possíveis e diversos impactos ocasionados nos pacientes e familiares.

\section{COMENTÁRIOS FINAIS}

É infrequente a ocorrência do complexo OEIS. O caso apresentado evidencia essa patologia através das características apresentadas pelo nosso paciente ao nascimento e relatada na revisão de literatura. Desse modo, é de fundamental importância seu diagnóstico pré-natal para o melhor manejo e consequente qualidade de vida desses pacientes.

\section{REFERÊNCIAS BIBLIOGRÁFICAS}

1. Carey JC, Greenbaum B, Hall BD. The OEIS complex (omphalocele, exstrophy, imperforate anus, spinal defects). Birth Defects; 1978. 14:253-63.

2. Hurwitz RS, Manzoni GAM, Ransley PG, Stephens FD. Cloacal exstrophy: a report of 34 cases. J Urol; 1987. 138: 1060-1064.

3. Yang F. OEIS Complex: A case report. J Diagnostic Med Sono; 2007. 23: 13-18.

4. Onkar, Deepali P. and Onkar, Prashant M. Omphalocele, exstrophy of bladder, imperforate anus and spinal defect (OEIS complex) - autopsy and prenatal ultrasound findings. Case Report. Eur. J. Anat. 2014. 18 (2): 123-127.

5. Chen CP, Shih SL, Liu FF, Jan SW, Jeng CJ, Lan CC. Perinatal features of omphaloceleexstrophy-imperforate anus-spinal defects(OEIS Complex) associated with large meningomyeloceles and severe limb defects. Am J Perinatol; 1997. 14: 275-279.

6. El-Hattab AW, SkorupskI JC, Hsieh MH, Breman AM, Patel A, Cheung SW, Craigen WJ. OEIS Complex associated with chromosome 1p36 deletion: A case report and review. Am J Med Genet, 2010. 152A: 504-511.

7. Chao, H S, Hsieh M, Lee H C. Multimodality Renal Failure in a Patient with OEIS Complex. Am J Perinatol Rep; 2015. 5:e161e164.

8. Goto S, Suzumori N, Obayashi S, Mizutani E, Hayashi Y, SugiuraOgasawara M. Prenatal findings of omphalocele-exstrophy of the bladder-imperforate anus-spinal defects (OEIS) complex. Congenit Anom (Kyoto). 2012. Sep;52(3):179-81.

9. Kaya M, Sancar S, Ozcakir E, Akdag A. Omphalocele, exstrophy of cloaca, imperforate anus and spinal defect. (OEIS Complex: A case report. Ped Uro Case Rep; 2015. 2(4):17-24.

10. Keppler-Noreuil KM, Gorton S, Foo F, Yankowitz J, Keegan C .Prenatal ascertainment of OEIS Complex/cloacal exstrophy-15 new cases and literature review. Am J Med Genet; 2007. 143A: 2122-2128.

11. Lubala TK, Shongo MY, Mbuyi SM, Mutombo AM, Ngwej DT, Kabange FN. OEIS Complex (omphalocele-exstrophy of the bladder-anal imperforation-spina bifida) and prenatal alcohol exposure: a case report. The Pan African Medical Journal;2013. 15:142. doi:10.11604/pamj.2013.15.142.2256

12. Patil NG, Mudanur SR, Nemagouda AS, Kori SS, Lahori LY. OEIS complex: a rare foetal anomaly. Int J Reprod Contracept Obstet Gynecol. 2014 Dec;3(4):1100-1103. 